\title{
Batería de Evaluación de Cognición Social en Psicología Forense: Adaptación y Validación
}

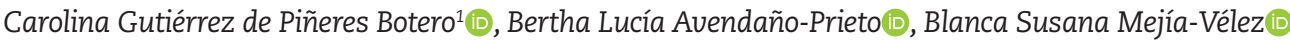 \\ Universidad Católica de Colombia, Bogotá, Colombia \\ Luz Anyela Morales-Quintero (D) \\ Universidad Autónoma de Puebla, Puebla, México \\ Ronald Toro (D) \\ Universidad Católica de Colombia, Bogotá, Colombia \\ Eric García-López \\ Instituto Nacional de Ciencias Penales, Ciudad de México, México
}

\section{RESUMEN}

El objetivo del estudio fue adaptar y validar una batería de instrumentos para evaluar subdominios de la cognición social con población forense en Colombia y México a partir de una selección de instrumentos utilizados en neuropsicología clínica. Se revisó la pertinencia de los subdominios que componen la cognición social como evidencia de validez de contenido mediante la evaluación de diferentes expertos en psicología forense, neuropsicología y medición y evaluación; utilizando el método ANGOFF modificado y un análisis de confiabilidad según el modelo Rasch y el coeficiente Alfa de Cronbach. Posteriormente fue sometido a pilotaje con 20 personas condenadas en cárceles colombianas y mexicanas con privación de libertad. Se encontraron calibraciones INFIT 1.17 y 1.04, OUTFIT 1.08 y 1.18 , con índices de separación de 2.66 y 1.63, y fiabilidad de .88 en colombianos y .73 en mexicanos; estos datos proporcionaron evidencias de validez de la batería adaptada.

Palabras clave: psicología forense; contexto penitenciario; cognición social; Angoff; Rasch.

RESUMO - Bateria de Avaliação para Cognição Social em Psicologia Forense: Adaptação e Validação

O objetivo do estudo foi adaptar e validar uma bateria para avaliar subdomínios da cognição social com população forense na Colômbia e no México a partir de uma seleção de instrumentos usados em neuropsicologia clínica. Revisou-se a relevância dos subdomínios que compõem a cognição social como evidência de validade de conteúdo por meio da avaliação de diferentes psicólogos especialistas em psicologia forense, neuropsicologia e medição e avaliação, usando o método ANGOFF modificado e uma análise de confiabilidade de acordo com o modelo de Rasch e coeficiente alfa de Cronbach. Posteriormente, ele foi submetido a pilotar com 20 pessoas privadas de liberdade sentenciadas em prisões colombianas e mexicanas. Calibrações INFIT 1,17 e 1,04, OUTFIT 1,08 e 1,18 foram encontradas, com taxas de separação de 2,66 e 1,63, uma confiabilidade de 0,88 em colombianos e 0,73 em mexicanos; esses dados forneceram evidências da validade da bateria adaptada.

Palavras-chave: psicologia forense; contexto prisional; cognição social; Angoff; Rasch.

\section{ABSTRACT - Assessment Battery for Social Cognition in Forensic Psychology: Adaptation and Validation}

The aim of the study was to adapt and validate a battery for the evaluation of subdomains of social cognition with the forensic population in Colombia and Mexico from a selection of instruments used in clinical neuropsychology. We reviewed the relevance of the subdomains that compose social cognition as evidence of content validity through the assessment of different psychologists, experts in forensic psychology, neuropsychology and measurement and evaluation, using the modified ANGOFF method and a reliability analysis according to the Rasch model and Cronbach's Alpha coefficient. Subsequently, a pilot study was performed with 20 people deprived of liberty in Colombian and Mexican prisons. INFIT calibrations of 1.17 and 1.04, and OUTFIT calibrations of 1.08 and 1.18, with separation rates of 2.66 and 1.63, and reliability of .88 and .73 were found in Colombians and Mexicans respectively; these data provide evidence of the validity of the adapted battery.

Keywords: forensic psychology; prison context; social cognition; Angoff; Rasch.

La cognición social se define como el conjunto de estructuras cerebrales y procesos cognitivos que favorecen la comprensión de las situaciones sociales y que median las reacciones conductuales ante ellas. Está integrada por una serie de subdominios que se ponen en marcha cuando las personas necesitan comprender lo que perciben, aprenden y recuerdan información de orden social, y favorecen la comprensión de señales informativas provenientes del entorno social, así como las decisiones, juicios, intenciones y comportamientos que resultan de la aplicación de estos procesos (Greifeneder et al., 2017; Quinn et al., 2006).

En general el concepto de cognición social y las definiciones de los sub dominios se encuentran en debate. 
Teóricamente algunos de ellos se sobreponen, pero los estudios con técnicas de neuroimagen muestran que los circuitos son diferentes aunque comparten algunas estructuras. Por ejemplo, Völlm et al. (2006) realizaron un estudio con resonancia magnética funcional con el fin de comparar las activaciones cerebrales en ambos procesos. Encontraron que que la Teoría de la Mente (TdM) y la empatía están asociados con redes neuronales superpuestas pero distintas.

Si bien no existe consenso sobre los diferentes sub dominios que integran la Cognición Social (CS) (Gourlay et al., 2020), algunos de los que tienen en la actualidad mayor consenso son: (a) Teoría de la Mente (TdM), que hace referencia a la capacidad para inferir estados mentales de los otros (creencias, metas, pensamientos, intenciones, entre otros) y construir una teoría sobre ellos (Baron-Cohen et al., 1985; Brüne, 2005), (b) la empatía, considerada como la capacidad para ser sensibles y comprender los estados sentimentales y afectivos de otras personas, sin que estos sean comunicados de manera explícita. Este subdominio se relaciona con la disposición para actuar a partir de esta comprensión (Baron-Cohen, 2009), (c) la percepción social que se refiere a la capacidad para identificar y valorar reglas y roles sociales, (d) el conocimiento social, entendido como la capacidad para identificar y hacer juicios sobre los aspectos sociales particulares de diversas situaciones (Addington et al., 2006), (e) el procesamiento emocional, definido como la capacidad para identificar, denominar, comprender, expresar y manejar emociones primarias y sociales a través del lenguaje verbal y no verbal (BaronCohen, 2009), y (f) el estilo atribucional como la manera en que cada persona explica las causas y consecuencias sobre diferentes situaciones sociales (Green et al., 2005; Penn et al., 2005).

Con el objetivo de lograr una evaluación directa y objetiva de los distintos subdominios de la CS, se han desarrollado una serie de instrumentos intentando suplir el tipo de exploración indirecta y subjetiva llevada a cabo tradicionalmente por psicólogos clínicos, especialmente en población diagnosticada con trastornos (Adenzato et al., 2010; Baron-Cohen, 2008; Book et al., 2007; Hastings et al., 2008; Lam et al., 2014; Pinkham et al., 2014; Vaskinn et al., 2015), así como en adultos sanos (Denburg et al., 2007; Denburg et al., 2005) que presentan alteraciones en uno o varios de los componentes de la cognición social.

Por esta razón, es plausible asumir que los diferentes instrumentos han sido diseñados, validados y utilizados con la población clínica, especialmente en personas con trastorno del espectro autista (Baron-Cohen, 2008; Morrison et al., 2019), psicosis y esquizofrenia (Lam et al., 2014; Pinkham et al., 2014; Pinkham et al., 2016; Pinkham et al., 2018), y personas con lesiones o daño cerebral (Adams et al., 2019: Adenzato et al., 2010; ShamayTsoory et al., 2003). Del mismo modo, se han diseñado instrumentos para la investigación de la CS, sin embargo, los instrumentos o baterías utilizados para la evaluación de este conjunto de procesos no ha sido incluido en la evaluación psicológica o neuropsicológica forense. Esto es de especial importancia si se tiene en cuenta la evidencia existente respecto a la relación entre cognición social, agresión y violencia (por ejemplo, Smeijers et al., 2020).

Al tener en cuenta en la selección de estos instrumentos que (a) fueran de libre acceso o que se tuviera el permiso de los autores para ser incluidos dentro de una batería viable para el contexto forense, además que incluyeran (b) material verbal y no verbal; que (c) hubieran sido validadas e incluidas en algún estudio previo con población latina, y que (d) evaluaran los diferentes subdominios de la CS, en la tabla 1 se presentan los subdominios y las pruebas correspondientes que pueden ser usados para conformar una batería de CS.

Al proponer una batería que permita evaluar la CS en el contexto forense y neuropsicológico, se responde a las necesidades del desarrollo de instrumentos psicométricos con las evidencias de validez necesarias en el campo. La psicología forense tiene el importante desafío de proveer instrumentos y conocimientos derivados de procedimientos objetivos (García-López et al., 2006) para brindar herramientas que puedan ser utilizadas en un ámbito en el que las exigencias son cada vez mayores (Morales, 2018). Los peritos en psicología y neuropsicología deben estar preparados para responder con fundamento a los cuestionamientos de las partes en litigio, así como defender sus conclusiones y la manera en que llegaron a ellas, al amparo de su experticia y de los desarrollos de su ciencia o disciplina (Gutiérrez de Piñeres et al., 2019). Además, sus argumentos han de presentarse de manera tan clara que los Órganos Jurisdiccionales puedan discernir sus aportes y la validez de los mismos para ser tomados en cuenta en las decisiones jurídicas (García-López \& Morales, 2017).

Desde esta perspectiva, los procesos de evaluación y con ello, la investigación enfocada en el desarrollo y adaptación de instrumentos útiles en el quehacer psicológico forense resultan prioritarios, en especial aquellos relacionados con la neuropsicología forense (Monroy \& García-López, 2017) De esta manera, el propósito de este estudio fue adaptar y validar un batería de cribado para evaluar la cognición social a nivel psicológico o neuropsicológico forense, en adultos vinculados a investigaciones en procesos judiciales o administrativos. 
Tabla 1

Subdominios y Pruebas Revisadas para el Desarrollo de una Batería de Cognición Social

\begin{tabular}{|c|c|c|}
\hline Subdominio & Prueba & Autores \\
\hline $\begin{array}{l}\text { Percepción social, } \\
\text { conocimiento social } \\
\text { y estilo atribucional }\end{array}$ & $\begin{array}{l}\text { - Comprensión de relaciones causales: } \\
\text { Identificación de Causas (1 estímulo de } \\
\text { ensayo, } 7 \text { reactivos) } \\
\text { - Comprensión de relaciones causales: } \\
\text { Identificación de Consecuencias (6 reactivos) } \\
\text { - Capacidad de juicio personal-social (11 } \\
\text { reactivos) } \\
\text { - Identificación de absurdos (6 reactivos) }\end{array}$ & $\begin{array}{l}\text { Hernández (2014) } \\
\text { Hernández y Yañez (2013) }\end{array}$ \\
\hline \multirow{3}{*}{$\begin{array}{l}\text { Teoría de la Mente } \\
\text { (TdM) }\end{array}$} & • FAUX PAS Adultos (20 reactivos) & Stone et al. (1998). \\
\hline & & $\begin{array}{l}\text { Traducción y adaptación al español } \\
\text { por Cecilia Serrano, del Hospital } \\
\text { Zubizarreta, Department of Neurology } \\
\text { and Neuropsychology, Buenos Aires, } \\
\text { Argentina. Adaptación al español Gil et } \\
\text { al. (2012). }\end{array}$ \\
\hline & $\begin{array}{l}\text { - Hinting Task (versión adaptada al español) } \\
\text { (10 reactivos) }\end{array}$ & $\begin{array}{l}\text { Corcoran et al. (1995). } \\
\text { Adaptación al español Gil et al. (2012). }\end{array}$ \\
\hline $\begin{array}{l}\text { Reconocimiento } \\
\text { emocional }\end{array}$ & Face Test (20 reactivos) & Baron-Cohen et al. (1997). \\
\hline Empatía & $\begin{array}{l}\text { Escala de Conducta de Cambridge (EQ) } \\
\text { (40 reactivos) }\end{array}$ & $\begin{array}{l}\text { Baron-Cohen y Wheelwright (2004); } \\
\text { Allison et al. (2011). } \\
\text { Adaptada al español por Pezzuto y } \\
\text { Fuentes (1999). }\end{array}$ \\
\hline
\end{tabular}

Fuente. Elaboración propia, a partir de Gutiérrez de Piñeres et al. (2019)

\section{Método}

\section{Participantes}

En la primera fase participaron nueve psicólogos forenses, quienes revisaron la pertinencia de los subdominios que componen la CS. En la segunda fase participaron 18 psicólogos, especialistas en psicología forense, neuropsicología y medición y evaluación, que respondieron un cuestionario acerca de los instrumentos utilizados en su práctica profesional para evaluar cada uno de los subdominios. Uno de los expertos era de nacionalidad argentina, uno de México y 16 colombianos. En cuanto al sexo, 16 eran de sexo femenino, 15 con nivel educativo de maestría, tres doctores, 12 manifestaron trabajar en el sector privado y 14 realizaban peritajes en diferentes instituciones. En la tercera fase, se convocó a 21 psicólogos (11 colombianos y 10 mexicanos), 17 psicólogos forenses, dos psicómetras, un psicólogo social y un psicólogo clínico, 19 con maestría y dos con estudios de doctorado. En la cuarta fase, se realizó el pilotaje con 20 personas con privación de la libertad, 10 colombianos cuya edad media era 36.6 años $(D E=5.76)$ y 10 mexicanos con una de edad media de 28.8 años $(D E=8.38)$. La escolaridad en los dos grupos se distribuyó de la siguiente forma: $25 \%$ con secundaria incompleta, $50 \%$ secundaria completa y $20 \%$ profesionales.

\section{Instrumentos}

Identificación de causas y consecuencias. Diseñada para evaluar la comprensión de relaciones causa-efecto, este instrumento mide la capacidad para inferir causas y consecuencias en una situación de interacción social. Está compuesta por 16 láminas (ocho causas -más una de ensayo- y ocho consecuencias), a partir de las cuales el evaluado debe explicar la acción más probable antes (causa) y después de la acción (consecuencia). Cada ítem se puntúa entre 0 y 2 basados en las instrucciones del manual original del instrumento, en el que aparece el puntaje por asignar desde una acción no probable a muy probable, los mayores puntajes indican una mayor capacidad para inferir causas y consecuencias en una acción. Así, se puntua 0 cuando la persona es incapaz de inferir la relación entre causa y efecto en una lámina determinada; 1 cuando la persona logra identificar este nexo, pero de forma incompleta; y 2, el puntaje más alto, cuando la persona identifica con claridad la relación causa-consecuencia.

Identificación de absurdos. Diseñada para evaluar las incongruencias en contextos sociales; está compuesta por seis láminas que contienen de cinco a ocho absurdos cada una, para un total de 37 reactivos, cada uno puntúa 0 si no detecta el absurdo o 1 si lo detecta. A mayores puntajes se asume una mayor capacidad 
para identificar incongruencias en un contexto social (Hernández \& Yáñez, 2013). En una evaluación realizada a adultos mayores en la Ciudad de México, se encontró un valor alfa de .80 , con valores de .67 para causas y .57 para consecuencias (Hernández, 2014).

Capacidad de juicio moral. Diseñada para evaluar la capacidad de una persona para comprender y generar soluciones a problemas en el ámbito social, que involucra un razonamiento de tipo moral; contiene 11 láminas temáticas sobre un problema social, cada una con una frase y una pregunta sobre un personaje, puntúa entre 0 y 2 (desde inconveniente o ilógica hasta correcta y segura). Los mayores puntajes indican mayor capacidad para generar soluciones a problemas en el ámbito personal o social (Hernández \& Yáñez, 2013).

Test de Caras - Face Test. Diseñado para evaluar la capacidad de las personas para reconocer estados emocionales a partir de las expresiones del rostro, contiene 20 fotografías con la cara de una mujer. En estas láminas se presentan fotografías que contienen 10 emociones básicas y 10 estados mentales complejos, en la parte inferior hay dos palabras que representan emociones, el evaluado debe elegir la que cree que mejor representa la imagen que está observando. Se puntúa 0 si no hay reconocimiento y 1 si hay reconocimiento. Los mayores puntajes indican mayor capacidad para identificar estados mentales básicos y complejos a través de material no verbal (Baron-Cohen et al., 1997).

Faux Paz o Test de Metida de Patas. Fue elaborada por Stone et al. (1998). Tiene como objetivo conocer si el evaluado tiene la capacidad de identificar si alguien ha dicho algo que no debió de manera imprudente, evalúa por lo tanto de forma indirecta empatía, en la medida en que quien responde las preguntas debe tener la capacidad de imaginar cómo se sintió afectada la persona sobre la que se estaba diciendo algo inadecuado, de manera negligente o descuidada (Butman, 2007). Está compuesta por 20 textos y preguntas referentes a estos. Los mayores puntajes en las historias que contienen un paso en falso, indican mayor capacidad para reconocer información a través del lenguaje indirecto. Se califican las historias que involucran una metida de pata, y aquellas de control en las que no existe una situación social inadecuada. En el estudio realizado por Gil et al., (2012) obtuvieron un coeficiente alfa de Cronbach de .64 para el grupo control y de .69 en un grupo de pacientes con esquizofrenia. La fiabilidad entre evaluadores estuvo por encima del .80 .

Hinting Task. Diseñado para evaluar la capacidad de las personas para inferir las intenciones reales detrás de las expresiones indirectas del habla (ironía o sarcasmo), está conformado por diez historias, en cada una aparecen dos personajes, al final uno de los personajes deja caer una indirecta bastante clara. Se pregunta ¿qué ha querido decir realmente? Cada historia se puntúa entre 0 y 2 , el 2 indica que la respuesta es correcta, 1 solicita ampliar información y 0 para respuestas incorrectas. El puntaje final en la prueba varía entre 0 y 20 (Gil et al., 2012). Los puntajes altos indican mayor capacidad para inferir estados mentales a través de material verbal.

Escala de Conducta de Cambridge. Cuestionario de autoinforme, diseñado para evaluar el Cociente de empatía (EQ), en adultos de inteligencia normal, cuyo objetivo es informar clínicamente, si el evaluado tienen dificultades en la empatía. En este instrumento no se separan los elementos en categorías puramente afectivas y cognitivas, debido a que, según sus autores, en la mayoría de los casos de empatía, los componentes afectivos y cognitivos coexisten y no se pueden aislar fácilmente (Baron-Cohen \& Wheelwright, 2004). El instrumento original contenía 60 items, 40 elementos de empatía y 20 elementos de relleno / control. Para esta batería se eligió la versión corta, de 40 reactivos, con formato de respuesta en escala Likert.

La prueba arroja puntuaciones entre 0 y 80 , resultados entre 0 y 32 indican un nivel bajo, valores entre 33 y 52 indican un nivel medio, puntajes entre 53 y 63 se consideran sobre la media y valores entre 64 y 80 indican niveles altos (Baron-Cohen \& Wheelwright, 2004). Esta escala ha sido aplicada a población forense mexicana con un índice de consistencia interna alfa de Cronbach .76 para personal encargado de la evaluación y tratamiento de menores infractores y .86 para personal de custodia y vigilancia en el mismo centro (Morales et al., 2017b).

\section{Procedimiento}

La investigación se realizó en cuatro fases. En la primera fase se determinaron los componentes de la CS, con la participación voluntaria de nueve psicólogos expertos en psicología forense y neuropsicología, quienes, a través de un cuestionario analizaron si los componentes de la CS pueden ser útiles en las evaluaciones realizadas en contextos forenses.

En la segunda fase 18 psicólogos forenses que no habían participado en la primera fase informaron sobre las pruebas que ellos en su práctica profesional aplicaban para evaluar cada uno de los subdominios de la CS, tanto en población menor de edad o adolescentes como en población adulta. Teniendo en cuenta los resultados de la anterior fase y con base en la batería adaptada por Hernández y Yáñez (2013), tres de los instrumentos diseñados por el Autism Research Centre, que facilitan el uso libre de sus test y permiten evaluar subdominios específicos de la CS y la adaptación al español del Hinting Task, se determinaron las pruebas que a juicio de estos expertos debían conformar la batería.

En la tercera fase se realizó la validez de contenido con 21 expertos en el tema, usando el método específico de Angoff Modificado, para evaluar la pertinencia, la suficiencia y la redacción de los ítems. Con los resultados obtenidos se revisó y ajustó la batería. Finalmente, en la cuarta etapa, previa firma del consentimiento informado, 
se realizó un pilotaje con un grupo de 20 personas privadas de la libertad, condenadas por delitos en una cárcel colombiana y una mexicana.

\section{Resultados y Discusión}

En la primera fase se determinó para cada uno de los reactivos, el CVR (Razón de validez de contenido) propuesto por Lawshe (Cohen \& Swerdlik, 2001), de lo cual se concluyó que en el $50 \%$ de los ítems se obtuvo un acuerdo perfecto entre los evaluadores. En cinco ítems que corresponden al 18\%, el índice obtenido fue de .78, valor aceptable para concluir acuerdo. Según Lawshe (1975), el valor mínimo de la razón de contenido para asegurar que es improbable que el acuerdo se deba al azar, cuando se tienen nueve expertos es .78, según este criterio, en el 68\% de los ítems se presentó acuerdo. En cuanto a la suficiencia, todos los jueces consideraron que el número de ítems era apropiado. De acuerdo con las calificaciones de los jueces y las observaciones realizadas a cada ítem, se hicieron las correcciones de cada afirmación del instrumento, de manera que la encuesta total quedó conformada por 25 ítems.
En la segunda fase, a partir de la información suministrada por los 18 jueces a través de la encuesta se conformó la batería de cribado con ocho instrumentos. En la tercera fase para realizar el análisis de los datos suministrados por los expertos (su valoración sobre cada una de las pruebas que componen la Batería), se utilizó el software Facets versión 3.80.2 (Linacre, 2016) que procesa la información con el Modelo de Rasch, este análisis proporcionó información sobre la consistencia del juicio realizado por los psicólogos forenses, clínicos y psicómetras, y permitió observar la pertinencia de la batería como un todo y en cada uno de sus instrumentos a partir de la valoración de los jueces expertos. En la tabla 2 se presenta el resumen de los datos, la media de los residuales fue de .00 para Colombia y -.01 para México y la media de los residuales estandarizados fue de .03 y - .02 para Colombia y México respectivamente, las desviaciones estándar poblacional y muestral fueron iguales a 1.04 para Colombia y 1.09 para México; se espera que la media de los residuales y de los residuales estandarizados tengan una puntuación de .00. Las desviaciones estándar (DE) se espera alcancen un valor de 1.00. El chi cuadrado para ambos países, presentó un ajuste global de los datos al modelo.

Tabla 2

Resumen de los Estadísticos Globales

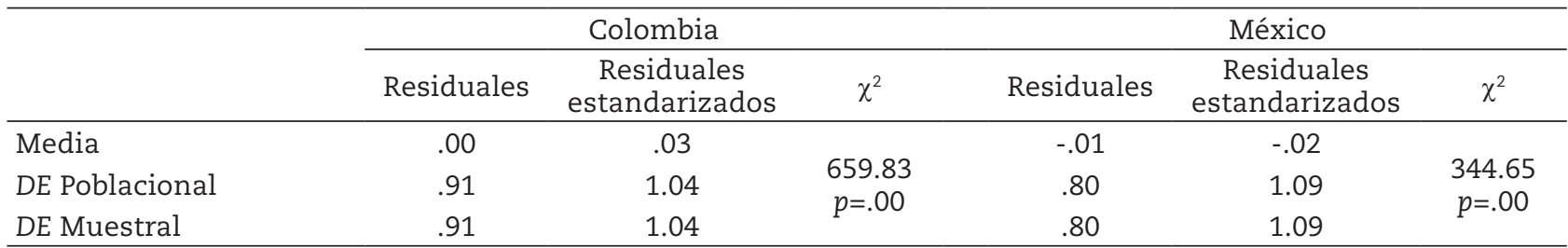

En la tabla 3 se muestra el resumen de los estadísticos tanto de los expertos como de los ítems. Para estimar el ajuste de los ítems se usan los parámetros INFIT y OUTFIT entre 0.8 y 1.3 logits, aunque un rango entre .40 y 1.40 logits es aceptable (González, 2008). Las medias del INFIT de los expertos e ítems para Colombia corresponden a 1.17 y 1.03 respectivamente, las medias para México fueron de 1.04 y 1.00 , la media del OUTFIT para Colombia, tanto de los expertos como de los ítems fue de 1.08 y para México fue de 1.18 (el ajuste perfecto equivale a 1.00). El índice de separación, es un indicador de la precisión con que se hicieron las valoraciones, este índice en los expertos colombianos fue de 2.66 junto con una fiabilidad de .88 , y en los expertos mexicanos fue de 1.63 con una frabilidad de .73 , los anteriores valores proporcionan una evidencia de validez (Wright \& Masters, 1982). Los índices de separación de los ítems valorados por los expertos colombianos y mexicanos se encontraron por debajo del mínimo aceptable (1.5), además, los valores de fiabilidad indicaron que los ítems deben revisarse para realizar los ajustes pertinentes.

El valor de severidad/indulgencia (Tabla 4), corresponde a la valoración que cada juez realizó de los ítems que componen cada prueba de la batería. El análisis del indicador anterior debe hacerse a la luz del ajuste próximo y el ajuste lejano. Los valores de ajuste indican el grado en que el desempeño de los expertos a lo largo de sus valoraciones se ajusta a la estimación del modelo. Según Linacre (2016) se espera un valor de ajuste menor o igual a 2.0; entre todos los datos que se presentan en la tabla 4 , se encuentra un solo caso en cada país con puntuaciones que no corresponden con lo esperado por el modelo (2.27 y 2.82 para el juez 11 de Colombia y 2.75 y 4.28 para el juez número 5 de México).

El siguiente indicador de la tabla 4, denominado correlación PtMea es una modificación del coeficiente punto biserial (PTBIS), y se interpreta como una correlación entre el puntaje particular observado por el juez y 
el puntaje global de todos los jueces. Para Colombia los puntajes de los jueces 5 y 11 no se correlacionaron con la puntuación global, la misma situación ocurre con los jueces 5 y 8 de México.

Tabla 3

Resumen de los Estadísticos de los Expertos y de los Ítems

\begin{tabular}{|c|c|c|c|c|}
\hline \multirow[b]{2}{*}{ Medidas } & \multicolumn{2}{|c|}{ Colombia } & \multicolumn{2}{|c|}{ México } \\
\hline & Expertos & Ítems & Expertos & Ítems \\
\hline$M$ & 9.14 & 9.14 & 9.55 & 9.55 \\
\hline$D E$ & .52 & .44 & .27 & .62 \\
\hline $\mathrm{N}$ & 11 & 29 & 10 & 29 \\
\hline Fiabilidad & .88 & .60 & .73 & .49 \\
\hline \multicolumn{5}{|l|}{ INFIT } \\
\hline M & 1.17 & 1.03 & 1.04 & 1.00 \\
\hline$D E$ & .53 & .70 & .69 & .55 \\
\hline \multicolumn{5}{|l|}{ OUTFIT } \\
\hline M & 1.08 & 1.08 & 1.18 & 1.18 \\
\hline$D E$ & .64 & 1.22 & 1.11 & 1.27 \\
\hline Índice de separación & 2.66 & 1.22 & 1.63 & .99 \\
\hline
\end{tabular}

Fuente. Elaboración propia

Tabla 4

Valores Obtenidos por los Evaluadores en el Juicio de Expertos

\begin{tabular}{|c|c|c|c|c|c|c|c|c|c|}
\hline \multicolumn{5}{|c|}{ Colombia } & \multicolumn{5}{|c|}{ México } \\
\hline Juez & $\begin{array}{l}\text { Severidad / } \\
\text { indulgencia }\end{array}$ & $\begin{array}{c}\text { Ajuste } \\
\text { próximo }\end{array}$ & $\begin{array}{l}\text { Ajuste } \\
\text { lejano }\end{array}$ & $\begin{array}{c}\text { Correlación } \\
\text { Ptmea }\end{array}$ & Juez & $\begin{array}{l}\text { Severidad / } \\
\text { indulgencia }\end{array}$ & $\begin{array}{c}\text { Ajuste } \\
\text { próximo }\end{array}$ & $\begin{array}{l}\text { Ajuste } \\
\text { lejano }\end{array}$ & $\begin{array}{c}\text { Correlación } \\
\text { Ptmea }\end{array}$ \\
\hline 7 & 8.03 & 1.38 & 1.30 & .43 & 6 & 8.93 & 1.29 & 1.11 & .66 \\
\hline 5 & 8.48 & .76 & .83 & .05 & 2 & 9.41 & .88 & .71 & .65 \\
\hline 6 & 8.86 & .65 & .63 & .40 & 3 & 9.48 & .60 & .87 & .59 \\
\hline 4 & 9.07 & 1.48 & 1.28 & .43 & 1 & 9.52 & .64 & .66 & .61 \\
\hline 1 & 9.24 & .39 & .42 & .55 & 7 & 9.52 & .66 & 1.17 & .62 \\
\hline 2 & 9.24 & .80 & .74 & .59 & 10 & 9.55 & .62 & .53 & .63 \\
\hline 3 & 9.24 & .96 & .86 & .60 & 4 & 9.55 & 1.57 & .62 & .52 \\
\hline 10 & 9.34 & 1.48 & 1.30 & .40 & 5 & 9.79 & 2.75 & 4.28 & .13 \\
\hline 11 & 9.59 & 2.27 & 2.82 & .28 & 9 & 9.79 & .52 & .69 & .49 \\
\hline 8 & 9.66 & 1.11 & .83 & .49 & 8 & 9.93 & .87 & 1.16 & .19 \\
\hline 9 & 9.83 & 1.59 & .90 & .34 & - & - & - & - & - \\
\hline
\end{tabular}

Fuente. Elaboración propia

La valoración dada por los expertos en cada uno de los ítems, presentados en la Tabla 5, arrojó promedios en Colombia entre 8.09 y 9.91, en México estas puntuaciones oscilaron entre 7.40 y 10 en una escala de 1 a 10 . Los ítems mejor evaluados por los expertos colombianos fueron los ítems 16, 15 y 14 que corresponden al Test de Caras y los ítems con más bajas puntuaciones fueron el 1 y el 5 que corresponden a la prueba de Comprensión de relaciones causales (identificación de causas) y Comprensión de relaciones causales (identificación de consecuencias), respectivamente. Los ítems mejor evaluados por los expertos mexicanos fueron los ítems 3, 7,
8, 13, 15, 16, 19 y 27 que obtuvieron la máxima puntuación y pertenecen a cinco de las seis pruebas evaluadas. Los ítems con más baja calificación fueron el ítem 10 y el ítem 9 que corresponden a la prueba de Capacidad de juicio personal-social. En esta escala un valor alto representa una mejor valoración por parte de los jueces.

En cuanto a los valores de ajuste próximo y lejano, la información de estimación se considera imprecisa si el valor supera 1.3, lo que indica ruido (aleatoriedad) en los datos y/o la presencia de valores extremos en las puntuaciones; situación que ocurrió con los ítems 13, 8, 17 y 4 para el caso de Colombia. El primero corresponde 
al test de caras, el ítem 8 corresponde a Comprensión de relaciones causales (identificación de consecuencias), el ítem 17 al Faux Pas y el ítem 4 a la prueba de Comprensión de relaciones causales (identificación de causas), situación que llevó a revisar las observaciones de los evaluadores, en particular en lo que hace referencia al aporte del instrumento al objetivo general de la batería (ítems 13 y 17) y a la pertinencia para la población objetivo (ítem 4 y 8 ).
Según los valores de ajuste próximo y lejano, los ítems evaluados por los expertos mexicanos que indican desajuste al modelo fueron los ítems 5, 6 y el 11. Los dos primeros corresponden a la prueba de comprensión de relaciones causales (identificación de consecuencias) y el ítem 11 evalúa la prueba de Capacidad de juicio personal-social. Para los primeros 8 ítems, estos valores no aplican, ya que todos los jueces los calificaron con el máximo puntaje. Los datos anteriores se presentan en la tabla 5.

Tabla 5

Valoración de la Pertinencia de Cada Ítem y Ajuste al Modelo de Rasch

\begin{tabular}{|c|c|c|c|c|c|c|c|}
\hline \multicolumn{4}{|c|}{ Colombia } & \multicolumn{4}{|c|}{ México } \\
\hline $\begin{array}{c}\text { Prueba/ } \\
\text { Ítem }\end{array}$ & $\mathrm{ME}$ & $\begin{array}{c}\text { Ajuste } \\
\text { próximo }\end{array}$ & $\begin{array}{l}\text { Ajuste } \\
\text { lejano }\end{array}$ & $\begin{array}{l}\text { Prueba/ } \\
\text { Ítem }\end{array}$ & $\mathrm{ME}$ & $\begin{array}{c}\text { Ajuste } \\
\text { próximo }\end{array}$ & $\begin{array}{l}\text { Ajuste } \\
\text { lejano }\end{array}$ \\
\hline Ítem 16 & 9.91 & .73 & .40 & Ítem 3 & 10.00 & NA & NA \\
\hline Ítem 15 & 9.73 & .80 & .49 & Ítem 7 & 10.00 & NA & NA \\
\hline Ítem 14 & 9.64 & .43 & .30 & Ítem 8 & 10.00 & NA & NA \\
\hline Ítem 24 & 9.55 & 1.23 & 1.00 & Ítem 13 & 10.00 & NA & NA \\
\hline Ítem 27 & 9.55 & .48 & .40 & Ítem 15 & 10.00 & NA & NA \\
\hline Ítem 11 & 9.45 & .86 & .59 & Ítem 16 & 10.00 & NA & NA \\
\hline Ítem 22 & 9.45 & .60 & .42 & Ítem 19 & 10.00 & NA & NA \\
\hline Ítem 23 & 9.45 & .76 & .52 & Ítem 27 & 10.00 & NA & NA \\
\hline Ítem 28 & 9.45 & .89 & .74 & Ítem 2 & 9.90 & .80 & .74 \\
\hline Ítem 13 & 9.36 & 3.82 & 6.85 & Ítem 4 & 9.90 & .80 & .74 \\
\hline Ítem 20 & 9.36 & 1.37 & 1.10 & Ítem 14 & 9.90 & .79 & .68 \\
\hline Ítem 7 & 9.27 & .50 & .52 & Ítem 1 & 9.80 & .70 & .59 \\
\hline Ítem 12 & 9.27 & .89 & .85 & Ítem 23 & 9.80 & .70 & .59 \\
\hline Ítem 25 & 9.27 & .65 & .69 & Ítem 25 & 9.80 & .81 & 1.22 \\
\hline Ítem 2 & 9.18 & 1.11 & 1.33 & Ítem 6 & 9.70 & 2.56 & 5.72 \\
\hline Ítem 3 & 9.18 & .64 & .67 & Ítem 28 & 9.70 & .82 & 2.15 \\
\hline Ítem 6 & 9.18 & .89 & .89 & Ítem 11 & 9.60 & 2 & .92 \\
\hline Ítem 21 & 9.18 & .67 & .61 & Ítem 18 & 9.60 & .47 & .35 \\
\hline Ítem 26 & 9.18 & .79 & .66 & Ítem 24 & 9.60 & .91 & .68 \\
\hline Ítem 19 & 9.09 & .82 & .68 & Ítem 5 & 9.50 & 2.13 & 3.70 \\
\hline Ítem 9 & 9.00 & .54 & .53 & Ítem 26 & 9.50 & .45 & .53 \\
\hline Ítem 8 & 8.91 & 1.60 & 1.92 & Ítem 29 & 9.50 & 1.11 & .89 \\
\hline Ítem 10 & 8.91 & 1.25 & .95 & Ítem 22 & 9.40 & .92 & .68 \\
\hline Ítem 18 & 8.91 & .85 & .89 & Ítem 12 & 9.30 & .82 & .50 \\
\hline Ítem 17 & 8.73 & 1.73 & 1.62 & Ítem 20 & 9.20 & .60 & .52 \\
\hline Ítem 4 & 8.27 & 2.61 & 2.18 & Ítem 17 & 9.00 & .64 & .96 \\
\hline Ítem 29 & 8.45 & 1.13 & 2.28 & Ítem 21 & 9.00 & 1.06 & .77 \\
\hline Ítem 5 & 8.18 & .64 & .63 & Ítem 10 & 7.80 & 1.14 & 1.07 \\
\hline Ítem 1 & 8.09 & .74 & .68 & Ítem 9 & 7.40 & .82 & .77 \\
\hline
\end{tabular}

$\mathrm{ME}=\mathrm{Media}$; $\mathrm{NA}=$ no aplica. Fuente. Elaboración propia

La neurociencia, la psicología y la neuropsicología forense, aportan conocimientos científicos para la toma de decisiones judiciales o administrativas (Anderson \& Kiehl, 2020; Freedman \& Zaami, 2019; van Dongen \& Franken, 2019), las cuales requieren evaluaciones, análisis e investigaciones altamente especializadas, confiables y con un nivel óptimo de objetividad (Bornstein \& Miller, 2016; Marcopulos et al., 2019). La Cognición
Social se ha incluido poco en este tipo de evaluaciones, y en ocasiones se utilizan instrumentos que no han sido adaptados ni a las poblaciones ni a los contextos forenses (Battaglia, 2020; Snowden \& Gray, 2010), o se evalúa sólo a través de la observación de comportamientos durante las entrevistas (Schmidt et al., 2015). Es por ello que se propuso la adaptación y validación de una batería, que permita evaluar el comportamiento social a 
través de la cognición social, con el fin de contribuir a mejorar la calidad de las de los informes y/o dictámenes realizados por psicólogos y neuropsicólogos forenses en su ejercicio profesional.

Adicionalmente a los análisis estadísticos y psicométricos realizados, se tuvieron en cuenta los comentarios incluidos por los expertos, al evaluar cada uno de los instrumentos, en los siguientes aspectos: 1 . Aporte del instrumento al objetivo general de la batería, 2. Relación del instrumento con uno o más objetivos específicos, 3. Competencia de los psicólogos forenses para aplicar la batería y 4. Pertinencia del instrumento para la población objetivo.

Las recomendaciones y comentarios generales dados por los evaluadores se resumen a continuación: incluir todos los ítems de la prueba y a partir del pilotaje determinar cuáles se pueden seleccionar, ya que se trata de una prueba de cribado. Además, la prueba piloto otorgará información relevante sobre aspectos culturales. Analizar las láminas que conformarán las diferentes pruebas en cuanto a su actualización.

Se considera pertinente indicar que es necesario (a) ofrecer entrenamiento a los psicólogos forenses y elaborar un protocolo de aplicación y calificación puesto que los contextos de reclusión pueden hacer engorrosa la aplicación, (b) tener en cuenta que la prueba Faux Pas requiere memoria, lo cual puede afectar los resultados, además, (c) debe ponerse límite de tiempo para la resolución de la batería; también, (d) es necesario establecer los criterios de calificación, (e) hacer una validación cognitiva y un análisis de contenido de respuestas verbales, y finalmente, (f) el Test de Cambridge no debe ser auto aplicado.

En la fase cuatro a partir de los anteriores resultados, sugerencias y recomendaciones de los expertos, se realizó el pilotaje para dar respuesta a los siguientes interrogantes: ¿Cómo se se puede controlar la deseabilidad social?, ¿Qué criterio puede utilizarse para medir el tiempo de aplicación? ¿Qué implica que una persona tarde más o menos'en dar respuesta a las pruebas? Teniendo en cuenta la aplicación piloto realizada, se propone para cada país una batería diferente. A continuación, se enuncian los cambios en las diferentes pruebas para cada país.
De la prueba de identificación de causas, compuesta inicialmente por siete láminas de respuesta y una de ensayo para Colombia se eliminaron dos: "futbol" porque genera confusión y "joven comiendo" porque no aporta a la consistencia interna. Para México, también se eliminó el ítem "futbol" y "lavar trastes" porque no aportaban a la consistencia interna. En la prueba de identificación de consecuencias, que inicialmente tenía siete láminas de respuesta y una de ensayo, en las dos baterías (Colombia y México), se eliminó el ítem "escribiendo carta" porque no aportaba a la consistencia interna. La prueba de identificación de absurdos, que inicialmente estaba compuesta por seis láminas, quedó igual para México, pero en la batería colombiana se eliminó la lámina de futbol porque no aportaba a la consistencia interna. En la prueba de capacidad de juicio personal social que contenía 15 láminas temáticas, se eliminaron dos en cada batería. En la batería colombiana no se incluyeron los ítems de "fila rápida" y "banco". En la batería mexicana no se incluyeron los ítems "super" y "viento", porque no aportaban a la consistencia interna, además en la lámina "super", los evaluados no comprendían que la situación hacía referencia al comportamiento del niño y respondían con referencia a otros actores. En el Test de caras (Face Test) de las 20 fotografías iniciales quedaron 18 en cada batería. En ambas se eliminó "caras 11" y de la batería para Colombia se eliminó "caras 15" y de la batería para México se eliminó “caras 8", además se cambiaron algunos adjetivos. La prueba Faux Pas compuesta por 20 textos y preguntas referentes a estos, quedó conformada por ocho (8) textos, cuatro (4) en los que alguien dijo algo que no debió decir y cuatro (4) de control, en las dos baterías quedaron los mismos textos. La Escala de Conducta de Cambridge no tuvo ningún cambio, los psicólogos que aplicaron las pruebas en el pilotaje recomendaron que no fuera auto aplicado por el nivel educativo de la población objeto de estudio. En la prueba Hinting Task o Test de las Insinuaciones conformada por diez historias, en la batería colombiana se eliminó la historia "regalo" y en la batería mexicana se eliminó la historia del "caramelo", porque no aportaban a la consistencia interna. La tabla 6 muestra el coeficiente alfa de Cronbach antes y después de los ajustes.

Tabla 6

Coeficientes Alfa de Cronbach Antes y Después de los Ajustes

\begin{tabular}{|c|c|c|c|c|}
\hline \multirow{3}{*}{ Pruebas } & \multicolumn{4}{|c|}{ Coeficiente Alfa de Cronbach } \\
\hline & \multicolumn{2}{|c|}{ Antes } & \multicolumn{2}{|c|}{ Después } \\
\hline & Colombia & México & Colombia & México \\
\hline Identificación de causas & .452 & .150 & .538 & .520 \\
\hline Identificación de consecuencias & .531 & .743 & .551 & .851 \\
\hline Identificación de absurdos & .511 & .600 & .584 & .600 \\
\hline Juicio personal social & .291 & .314 & .457 & .636 \\
\hline Face Test & .432 & .007 & .567 & .433 \\
\hline Faux Pas & .833 & .873 & .833 & .873 \\
\hline Hinting Task & .589 & .524 & .628 & .728 \\
\hline
\end{tabular}

Fuente. Elaboración propia 


\section{Consideraciones finales}

A través de todas las fases de la investigación la batería fue validada y ajustada según las recomendaciones y sugerencias de los evaluadores. Los resultados de los estadísticos de las evaluaciones realizadas por los expertos colombianos y mexicanos evidenciaron un ajuste global de los datos al modelo. Según los indicadores INFIT y OUTFIT de los expertos de los dos países se puede concluir que la evaluación realizada por ellos fue de alta calidad. Los valores de fiabilidad de los ítems de ambos países indicaron que los ítems deben revisarse y realizar los ajustes pertinentes a los instrumentos.

En general todos los expertos evaluaron con puntajes altos los instrumentos de la Batería, encontrándose las puntuaciones más bajas en la evaluación de la prueba de Comprensión de relaciones causales (identificación de causas), Comprensión de relaciones causales (identificación de consecuencias) y Capacidad de juicio personal-social. De acuerdo con las recomendaciones realizadas por los expertos, se elaboraron protocolos de aplicación para los instrumentos, de manera que los psicólogos forenses cuentan con una guía tanto de aplicación como de calificación.

Finalmente, con base en las evaluaciones y observaciones de los 48 psicólogos que participaron en las tres fases del estudio y el pilotaje realizado con los 20 participantes privados de la libertad, se conformó una batería de cribado, que puede ser utilizada en población mayor de 18 años, colombiana y mexicana de manera gratuita, siempre y cuando se reconozcan los derechos de autor.

De acuerdo con la validación realizada con expertos en el ámbito forense, la CS constituye una variable relevante en estos contextos, en concordancia con la evidencia sobre la relación entre ésta, la agresión, la violencia y el comportamiento delictivo violento (Garofalo et al., 2018; Smeijers et al., 2020). Por lo tanto, es necesario contar con instrumentos que permitan conocer la CS en poblaciones forenses, no sólo con fines de evaluación y realización de peritajes, sino también para monitorear los cambios que se puedan atribuir a los programas dirigidos a modificar la CS en grupos vinculados con el Sistema de Justicia, por ejemplo, en diferentes tipos de delincuentes que cumplen una sentencia. En este sentido, la batería propuesta constituye un avance importante para la evaluación de la CS en población forense.

El presente estudio contó con algunas limitaciones: todos los participantes eran de sexo masculino, la muestra obtenida para la prueba piloto fue reducida y no se consideró el tipo de delito cometido, aspectos que impiden generalizar los hallazgos. Se sugiere para futuros estudios repetir el análisis psicométrico con una muestra más amplia que incluya controles y otras poblaciones que podrían ser parte de evaluaciones psicológicas y neuropsicológicas forenses. Del mismo modo se sugiere realizar una validación ecológica, realizar análisis factoriales para la Escala de Conducta de Cambridge, y obtener baremos para diferentes poblaciones.
La batería de CS presentada es útil para su uso en psicología y neuropsicología forenses. Es una batería breve, cuya aplicación toma aproximadamente 30 minutos. Está conformada por un conjunto de instrumentos de fácil aplicación y calificación, que orientan para tomar una decisión de realizar una evaluación de CS más amplia, de acuerdo con los resultados obtenidos en cada sub-prueba. La batería final quedó conformada por cuatro subdominios y ocho subpruebas que mostraron buenas propiedades psicométricas. Este es un aporte relevante, especialmente en Hispanoamérica, donde existen pocas pruebas validadas para los contextos forenses.

\section{Agradecimientos}

Los autores agradecen a la Universidad Católica de Colombia por su apoyo financiero en el desarrollo del proyecto de investigación y los productos derivados.

\section{Financiación}

Todas las fuentes de financiación para la preparación y producción del estudio (recopilación de datos, análisis e interpretación, así como la redacción de los resultados en el presente en el manuscrito) fueron proporcionadas por el proyecto de investigación "Validación de Contenido de una Batería de Cognición Social en Psicología Forense con Población Colombiana y Población Mexicana, Código del proyecto: 4110003, Universidad Católica de Colombia.

\section{Contribuciones de los autores}

Declaramos que todos los autores participaron en la preparación del manuscrito. En concreto, los autores Carolina Gutiérrez de Piñeres Botero, Bertha Lucía Avendaño-Prieto Luz Anyela Morales-Quintero y Eric García-López participaron en la redacción inicial del estudio - conceptualización, investigación, visualización, los autores Bertha Lucía Avendaño-Prieto, Blanca Susana Mejía-Vélez, Luz Anyela Morales-Quintero y Ronald Toro participaron en el análisis de datos, y los autores Carolina Gutiérrez de Piñeres Botero, Bertha Lucía Avendaño-Prieto, Blanca Susana Mejía-Vélez, Luz Anyela Morales-Quintero y Ronald Toro participaron en la redacción final del trabajo - corrección y edición.

\section{Disponibilidad de datos y materiales}

Todos los datos y sintaxis generados y analizados durante esta investigación serán tratados en completo secreto debido a los requerimientos del Comité de Ética en Investigación en Seres Humanos. Sin embargo, el conjunto de datos y sintaxis que respaldan las conclusiones de este artículo están disponibles previa solicitud razonable al autor principal del estudio

\section{Conflicto de intereses}

Los autores declaran no tener ningún conflicto de intereses. 


\section{Referencias}

Adams, A.G., Schweitzer, D., Molenberghs, P. \& Henry, J.D. (2019). A meta-analytic review of social cognitive function following stroke. Neuroscience and Biobehavioral Reviews, 102, 400-416. https://doi.org/10.1016/j.neubiorev.2019.03.011

Addington, J., Saeedi, H., \& Addington, D. (2006). Influence of social perception and social knowledge on cognitive and social functioning in early psychosis. The British Journal of Psychiatry, 189(4), 373-378. https://doi.org/10.1192/bjp.bp.105.021022

Adenzato, M., Cavallo, M., \& Enrici, I. (2010). Theory of Mind ability in the behavioural variant of frontotemporal dementia: An analysis of the neural, cognitive and social levels. Neuropsychologia, 48(1), 2-12. https://doi.org/10.1016/j.neuropsychologia.2009.08.001.

Allison, C., Baron-Cohen, S., Wheelwright, S. J., Stone, M. H., \& Muncer, S. J. (2011). Psychometric analysis of the Empathy Quotient (EQ). Personality and Individual Differences, 51(7), 829-835. https://doi.org/10.1016/j.paid.2011.07.005

Anderson, N. E. \& Kiehl, K. E. (2020). Re-wiring Guilt: How Advancing Neuroscience Encourages Strategic Interventions Over Retributive Justice. Research Topic, Neurolaw: The Call for Adjusting Theory Based on Scientific Results. Frontiers in Psychology, 11, 1-12. https:// doi.org/10.3389/fpsyg.2020.00390

Baron-Cohen, S. (2008). Autism, hypersystemizing, and truth. Quarterly Journal of Experimental Psychology (Hove), 61(1), 64-75. https://doi. org/10.1080/17470210701508749

Baron-Cohen, S. (2009). Autism: The Empathizing-Systemizing (E-S) Theory. Annals of the New York Academy of Sciences, 11(56), 68-80. https://doi.org/10.1111/j.1749-6632.2009.04467.x.

Baron-Cohen, S., \& Wheelwright, S. (2004). The empathy quotient: an investigation of adults with Asperger syndrome or high functioning autism, and normal sex differences. Journal of Autism and Developmental Disorders, 34(2), 163-175. https://doi.org/10.1023/ B:JADD.0000022607.19833.00

Baron-Cohen, S., Leslie, A. M., \& Frith, V. (1985). Does the autistic child have a 'theory of mind'? Cognition, 21(1), 37-46. https://doi. org/10.1016/0010-0277(85)90022-8

Baron-Cohen, S., Wheelwright, S., \& Jolliffe, T. (1997). Is there a "language of the eyes"? Evidence from normal adults, and adults with autism or Asperger syndrome. Visual Cognition, 4(3), 311-331. https://doi.org/10.1080/713756761

Baron-Cohen, S., \& Wheelwright, S. (2004). The empathy quotient: an investigation of adults with Asperger syndrome or high functioning autism, and normal sex differences. Journal of Autism and Developmental Disorders, 34(2), 163-175. https://doi.org/10.1023/ b:jadd.0000022607.19833.00

Battaglia, A. (2020). Social Cognition and Aggression in Forensic Psychiatric Patients. [Masters dissertation, York University - Ontário]. Disponible en http://hdl.handle.net/10315/37771

Book, A. S., Quinsey, V. L., \& Langford, D. (2007). Psychopathy and the perception of affect and vulnerability. Criminal Justice and Behavior, 34(4), 531-544. https://doi.org/10.1177/0093854806293554

Bornstein, B. H., \& Miller, M. K. (Eds.). (2016). Advances in psychology and law. New York: Springer International Publishing

Brüne, M. (2005). Emotion recognition, 'theory of mind' and social behaviour in schizophrenia. Psychiatry Research, 133(2-3), 135-147. https://doi.org/10.1016/j.psychres.2004.10.007

Butman, J. (2007). Evaluación de la Cognición Social. En: Burin, D., Drake, M., y Harris, P. Evaluación Neuropsicológica en Adultos (pp. 331343). Buenos Aires: Paidós.

Cohen, R., \& Swerdlik, S. (2001). Pruebas y evaluación psicológicas. Introducción a las pruebas y a la medición (4ta. ed.). México: Mc Graw Hill.

Corcoran, R., Mercer, G., \& Frith, C. D. (1995). Schizophrenia, symptomatology and social inference: investigating "theory of mind" in people with schizophrenia. Schizophrenia Research, 17(1), 5-13. https://doi.org/10.1016/0920-9964(95)00024-G

Denburg, N., Cole, C., Hernández, M., Yamada, T., Tranel, D., Bechara, A., \& Wallace, R. (2007). The orbitofrontal cortex, real-world decision making and normal aging. Annals of New York Academy Sciences, 11(21), 480-498. https://doi.org/10.1196/annals.1401.031

Denburg, N. L., Tranel, D., \& Bechara, A. (2005). The ability to decide advantageously declines prematurely in some normal older persons. Neuropsychologia, 43(7), 1099-1106. https://doi.org/10.1016/j.neuropsychologia.2004.09.012

Freedman, D. \& Zaami, S. (2019). Neuroscience and mental state issues in forensic assessment. International Journal of Law and Psychiatry 65, 101437. https://doi.org/10.1016/j.ijlp.2019.03.006

García-López, E., \& Morales, L.A. (2017). Psicopatología Forense en México. En Z. García y M. E. Bravo (Eds). El estado del arte de las ciencias forenses en México (pp. 287-312). México: Tirant lo blanch.

García-López, E., Pérez, A., \& Lacalle, J. (2006). Psicología jurídica-forense y juicios orales en materia penal: perspectivas, riesgos y desafíos. Jus Semper Loquitur, 50(2), 23-32.

Garofalo, C., Velotti, P., \& Zabattini, G.C. (2018). Emotion regulation and aggression: The incremental contribution of alexithymia, impulsivity and emotion dysregulation facets. Psychology of Violence, 8(4), 470-483. https://doi.org/10.1037/vio0000141

Gil, D., Fernández-Modamio, M., Bengochea, R., \& Arrieta, M. (2012). Adaptación al español de la prueba de teoría de la mente Hinting Task. Revista de Psiquiatría y Salud Mental, 5(2), 79-88. https://doi.org/10.1016/j.rpsm.2011.11.004

González, M. (2008). El Análisis de Reactivos con el Modelo Rasch. Manual Técnico A. Serie: Medición y Metodología, Universidad de Sonora, México. Disponible en http://www.winsteps.com/a/recursos-offline.pdf

Gourlay, C., Collin, P., Caron, P., DÁuteuil, C., \& Scherzer, P.B. (2020). Psychometric assessment of social cognitive tasks. Applied Neuropsychology: Adult. Online first, 1-19 https://doi.org/10.1080/23279095.2020.1807348

Green, M. F., Olivier, B., Crawley, J. N., Penn, D. L., \& Silverstein, S. (2005). Social cognition in schizophrenia: recommendations from the measurement and treatment research to improve cognition in schizophrenia new approaches conference. Schizophrenia Bulletin, 31(4), 882-887. https://doi.org/10.1093/schbul/sbi049

Greifeneder, R., Bless, H., \& Fiedler, K. (2017). Social cognition: How individuals construct social reality. Psychology Press.

Gutiérrez de Piñeres, C., Avendaño, B., Mejía, S., Morales, L., \& García-López, E. (2019). Evaluación Neuropsicológica Forense de la Cognición Social. México: Manual Moderno.

Hastings, M. E., Tangney, J. P., \& Stuewig, J. (2008). Psychopathy and identification of facial expressions of emotion. Personality and individual differences, 44(7), 1474-1483. https://doi.org/10.1016/j.paid.2008.01.004.

Hernández, A. (2014). Evaluación de la cognición social en adultos mayores de la Ciudad de México [Tesis doctoral, Universidad Autónoma de México - México, D.F].

Hernández, A., \& Yáñez, M. G. (2013). Evaluación de la Cognición Social en Adultos Mayores: Presentación de la batería COGSOC-AM. Revista Argentina de Clínica Psicológica, 22(3), 269-278. Available at https://www.redalyc.org/pdf/2819/281935590007.pdf

Morrison, K. E., Pinkham, A. E., Kelsven, S., Ludwieg, K., Penn, D. L. \& Sasson, N. J. (2019). Psychometric evaluation of social cognitive measures for adults with autism. Autism Research, 12(5), 766-778. https://doi.org/10.1002/aur.2084 
Lam, B. Y., Raine, A., \& Lee, T. M. (2014). The relationship between neurocognition and symptomatology in people with schizophrenia: social cognition as the mediator. BMC Psychiatry, 14(1), 1-10. https://doi.org/10.1186/1471-244X-14-138.

Lawshe, C. H. (1975). A quantitative approach to content validity. Personnel Psychology, 28(4), 563-575.

Linacre, J.M. (2016). Winsteps ${ }^{\circledR}$ (Version 3.92.0) [Computer Software]. Beaverton, Oregon: Winsteps.com. Available at http://www.winsteps. com/Messick

Marcopulos B.A., Welner M., Campbell K.T. (2019) Forensic Challenges in Medical Settings for Physicians and Neuropsychologists. En: K. Sanders (eds). Physician's Field Guide to Neuropsychology (pp. 495-506). Springer, New York, NY. https://doi.org/10.1007/978-1-49398722-1_29

Monroy, N. \& García-López, E. (2017). Neuropsicología forense, una aportación al sistema de justicia en México. Revista Criminalidad, 59(3), 163-181. Disponible en http://www.scielo.org.co/pdf/crim/v59n3/1794-3108-crim-59-03-00163.pdf

Morales, L. A. (2018). La prueba y su valoración en México: implicaciones para el quehacer criminológico. En V. Garrido (Comp.) Tratado de Criminología Forense I. La Criminología Forense y el Informe Criminológico (pp. 238-308). Ciudad de México: Tirant lo blanch.

Penn, D., Roberts, D. L., Munt, E. D., Silverstein, E., Jones, N., \& Sheitman, B. (2005). A pilot study of social cognition and interaction training (SCIT) for schizophrenia. Schizophrenia research, 80(2), 357-359. https://doi.org/10.1016/j.schres.2005.07.011

Pezzuto, C., \& Fuentes, J. (1999). Empathy Quotient (EQ) - Español. Autism Research Center, University of Cambridge. Available at https:// www.autismresearchcentre.com/arc_tests

Pinkham, A. E., Penn, D. L., Green, M. F., Buck, B., Healey, K., \& Harvey, P. D. (2014). The Social cognition psychometric evaluation study: Results of the expert survey and RAND panel. Schizophrenia Bulletin, 40(4), 813-823. https://doi.org/10.1093/schbul/sbt081

Pinkham, A. E., Penn, D. L., Green, M. F., \& Harvey, P. D. (2016). Social cognition psychometric evaluation: Results of the initial psychometric study. Schizophrenia Bulletin, 42(2), 494-504. https://doi.org/10.1093/schbul/sbv056.

Pinkham, A.E., Harvey, Ph.D., Penn, D. L. (2018). Social cognition psychometric evaluation. Results of the final validation study. Schizophrenia Bulletin, 44(4), 737-748. https://doi.org/10.1093/schbul/sbx117

Quinn, K. A., Macrae, C. N., \& Bodenhausen, G. V. (2006). Social cognition. Encyclopedia of cognitive science. https://doi.org/10.1002/0470018860. s00619.

Shamay-Tsoory, S. G., Tomer, R., Berger, B. D., \& Aharon-Peretz, J. (2003). Characterization of empathy deficits following prefrontal brain damage: the role of the right ventromedial prefrontal cortex. Journal of Cognitive Neuroscience, 15(3), 324-337. https://doi. org/10.1162/089892903321593063

Schmidt, A. F., Banse, R., \& Imhoff, R. (2015). Indirect Measures in Forensic Contexts. In T. M. Ortner \& F. J. R. van de Vijver (Eds.). Behavior-Based Assessment: Going Beyond Self-Report in the Personality, Affective, Motivation, and Social Domains (pp. 173-194). Göttingen: Hogrefe.

Smeijers, D., Benbouriche, M., \& Garofalo, C. (2020). The association between emotion, social information processing, and aggressive behaviour: A systematic review. European Psychologist, 25(2), 81-91. https://doi.org/10.1027/1016-9040/a000395

Snowden, R. J., \& Gray, N. S. (2010). Implicit social cognition in forensic settings. In B. Gawronski \& B. K. Payne (Eds.), Handbook of implicit social cognition: Measurement, theory, and applications (pp. 522-534). NY: The Guilford Press.

Stone, V. E., Baron-Cohen, S., \& Knight, R. T. (1998). Frontal lobe contributions to theory of mind. Journal of Cognitive Neuroscience, 10(5), 640-656. https://doi.org/10.1162/089892998562942

van Dongen, J. D., \& Franken, I. H. (2019). Neuroscience in forensic psychiatry and psychology: An introduction to the special issue. International Journal of Forensic Mental Health, 18(3), 179-186. https://doi.org/10.1080/14999013.2019.1652708

Vaskinn, A., Antonsen, B. T., Fretland, R. A., Dziobek, I., Sundet, K., \& Wilberg, T. (2015). Theory of mind in women with borderline personality disorder or schizophrenia: differences in overall ability and error patterns. Frontiers in Psychology, 6, 1-7. https://doi. org/10.3389/fpsyg.2015.01239

Völlm, B. A., Taylor, A. N. W., Richardson, P., Corcoran, R., Stirling, J., McKie, S., ... Elliott, R. (2006). Neuronal correlates of theory of mind and empathy: A functional magnetic resonance imaging study in a nonverbal task. NeuroImage, 29(1), 90-98. https://doi. org/10.1016/j.neuroimage.2005.07.022

Wright, B. D., \& Masters, G. N. (1982). Rating Scale Analysis. Chicago: MESA Press, 1982.

recebido em janeiro de 2019 aprovado em outubro de 2020

\section{Sobre os autores}

Carolina Gutiérrez de Piñeres Botero es Doctora en Psicología con Orientación en Neurociencia Cognitiva Aplicada de la Universidad de Maimónides, es actual Docente del programa de Doctorado de la Universidad Católica de Colombia.

Bertha Lucía Avendaño-Prieto es Doctora en Psicología de la Universidad de la Laguna, es actual Docente del programa de Doctorado de la Universidad Católica de Colombia.

Blanca Susana Mejía-Vélez es licenciada en Estadística y Magister en Evaluación en Educación. Es docente en las áreas de Metodología de Investigación y Estadística de la Universidad Católica de Colombia.

Luz Anyela Morales-Quintero es Doctora en Psicología Social por la Universidad Autónoma de Madrid (España), es profesorainvestigadora en el Programa de Criminología de la Facultad de Derecho y Ciencias Sociales de la Benemérita Universidad Autónoma de Puebla.

Ronald Alberto Toro Tobar es Psicólogo Clínico, Magister en Psicología, doctorando en Salud, Psicología y Psiquiatría de la Universidad de Almería. Actualmente, es docente investigador de la Universidad Católica de Colombia.

Eric García-López es Doctor en Psicopatología Forense y periodo docente del Doctorado en Neurociencia por la Universidad Complutense de Madrid. Postdoctorado en Evolución y Cognición Humana IFISC-UIB. Investigador titular en el Instituto Nacional de Ciencias Penales.

\section{Como citar este artigo}

Botero et al. (2021). Batería de Evaluación de Cognición Social en Psicología Forense: Adaptación y Validación. Avaliação Psicológica, 20(1), 89-99. http://dx.doi.org/10.15689/ap. 2021.2001.17545.10 